\title{
CALCULATION OF RUIN PROBABILITIES WHEN THE CLAIM DISTRIBUTION IS LOGNORMAL
}

\author{
Olof Thorin and NiLs Wikstad \\ Stockholm \\ SUMMARY
}

In this paper some ruin probabilities are calculated for an example of a lognormal claim distribution. For that purpose it is shown that the lognormal distribution function, $\Lambda(y)$, may be written in the form

$$
\Lambda(y)=\int_{0}^{\infty}\left(1-e^{-x y}\right) d V(x)
$$

where $V(x)$ is absolutely continuous and without being a distribution function preserves some useful properties of such a function.

An attempt is also made to give an approximant $\Lambda_{a}(y)$ to $\Lambda(y)$ such that $\Lambda_{a}(y)$ is a linear combination of a low number of exponential distributions. For comparison, ruin probabilities are also calculated for two examples of $\Lambda_{a}(y)$.

In the considered numerical cases it is assumed that the occurrence of claims follows a Poisson process.

\section{INTRODUCTION}

This paper can be viewed as a continuation of our previous joint paper (Thorin and Wikstad (I973)). In that paper we made numerical evaluations of ruin probabilities when the distribution functions of the amounts of claims, $P(y)$, and of the interclaim times, $K(t)$, both could be expressed as a weighting together of exponential distributions. In fact we considered ${ }^{1}$ ) the following two classes

$$
P(y)= \begin{cases}\int_{0}^{\infty}\left(1-e^{-x y}\right) d V(x), & y \geqq 0 \\ 0, & y<0\end{cases}
$$

1) As to the class (I.I) we referred to Seal (I969). However, we should also have referred to Thynon (I964) where a systematic study of the class (I.I) 1.a. including the Pareto example was given. 


$$
K(t)= \begin{cases}\int_{0}^{\infty}\left(\mathrm{I}-e^{v t}\right) d W(v), & t \geqq 0 \\ 0, & t<0\end{cases}
$$

where $V(x)$ and $W(v)$ were distribution functions such that $V(0)=W(0)=0$.

Besides the simples cases when $V(x)$ and $W(v)$ are discrete distributions with a finite number of spikes we also considered absolutely continuous $V(x)$ or $W(v)$. In particular, we gave formulas and numerical values of the ruin probabilities when $V(x)$ (or $W(v)$ ) was a $\Gamma$-distribution corresponding to a Pareto distribution for $P(y)(K(t))$. For that case we also gave an approximant with a finite number of spikes. It turned out that the ruin probabilities were well approximated for moderate values of the initial risk reserve. For large values of the initial reserve, however, discrepancies appeared corresponding to entirely different asymptotic behaviors.

In the present paper we attempt to generalize our procedures to a case where $V(x)$ no longer is a distribution function but still satisfies the conditions:

(i) $V(0)=0, V(\infty)=I$

(ii) $V(x)$ is right-continuous

(iii) $\int_{0}^{\infty}|d V(x)|<\infty$, i.e. $V(x)$ is of bounded variation over the entire interval $(0, \infty)$.

Of course, not every such $V(x)$ inserted in formula (I.I) gives a $P(y)$ which is a distribution function. However, in certain cases we get a distribution function. Let us first take a simple example. We let

$$
V(x)=a \varepsilon\left(x-\alpha_{1}\right)+(I-a) \varepsilon\left(x-\alpha_{2}\right)
$$

where $0<\alpha_{1}<\alpha_{2}, a=\alpha_{2} /\left(\alpha_{2}-\alpha_{1}\right)$.

The second weight $\mathrm{I}-a=-\alpha_{1} /\left(\alpha_{2}-\alpha_{1}\right)$ is thus negative, Inserting $V(x)$ in formula (I.I) we "get

$$
\begin{aligned}
P(y) & =\mathrm{I}-\frac{\alpha_{2}}{\alpha_{2}-\alpha_{1}} e^{-\alpha_{1} y}+\frac{\alpha_{1}}{\alpha_{2}-\alpha_{1}} e^{-\alpha_{2} y}= \\
& =\left(\mathrm{I}-e^{-\alpha_{1} y}\right) *\left(\mathrm{I}-e^{-\alpha_{2} y}\right)
\end{aligned}
$$


i.e. the convolution of two simple exponential distributions. In passing, we note the obvious fact that for $\dot{V}(x)$ in (I.3) the first moment is zero and all the higher moments are negative. (This fact has an obvious generalization to convolutions of $n$ exponential distributions.)

As the reader easily realizes there is an abundance of such examples as (I.3) where a finite number of spikes, among them some negative ones, produce distribution functions $P(y)$. The same can, of course, be said about $K(t)$. The numerical problem of calculating ruin probabilities in such cases present no essential difficulties as compared with the cases where $V(x)$ and $W(v)$ consist of only positive spikes.

The main topic of this paper is, however, a case where $V(x)$, without being a distribution function, is absolutely continuous and, in fact, produces the lognormal distribution $\Lambda(y)$ for $P(y)$. For a special parameter choice we attempt to calculate a number of ruin probabilities and also, for comparison, to bring forward and determine ruin probabilities for an approximant $\Lambda_{a}(y)$ to $\Lambda(y)$ such that the corresponding $V_{a}(x)$ consists of a low number of spikes, which if necessary may contain negative ones. As to $K(t)$ our formulas are general. However, for numerical purposes we consider only the case $K(t)=\mathrm{I}-e^{-t}$, i.e. we assume that the occurrence of claims obeys a Poisson process.

In section 2 we consider the function $V(x)$ producing the lognormal distribution. Thereafter, the section 3 gives the formulas for the ruin probabilities. Section 4 treats the principles for obtaining $\Lambda_{a}(y)$. In section 5 the asymptotic behaviour of $\Psi(u)$ for $u \rightarrow \infty$ is dealt with where $\Psi(u)$ denotes the ruin probability for an infinite time when the initial risk reserve is $u$. Section 6 presents the numerical methods. Finally, section 7 and the attached tables give the numerical results. Section 8 contains some concluding remarks.

2. The Function $V(x)$ Producing the Lognormal Distribution, $\Lambda(y)$

The lognormal distribution function, $\Lambda(y)$, has the well known form

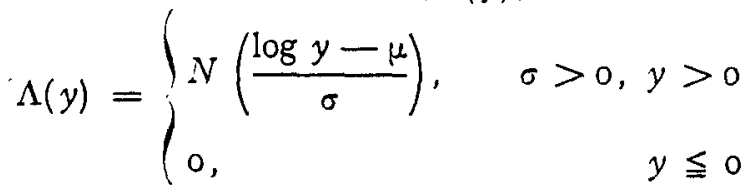


where $N(\cdot)$ stands for the normal distribution function with mean zero and variance one, i.e.,

$$
N(y)=\frac{I}{\sqrt{2 \mu}} \int_{-\infty}^{y} e^{-v^{2} / 2} d v
$$

and $\log$ denotes the natural logarithm. As a general reference for the lognormal distribution see Aitchison and Brown (r957).

For convenience, we introduce $\alpha=e^{-\mu}$ and use $\beta$ instead of $\sigma$. Thus

$$
\Lambda(y)= \begin{cases}N\left(\frac{\log (\alpha y)}{\beta}\right), \alpha>0, \beta>0, & y>0 \\ 0, & y \leqq 0\end{cases}
$$

Clearly, the parameter $\alpha$ is a pure scale parameter in the same sense as $\alpha$ in $F(y)=\mathrm{I}-e^{-x y}, y \geqq 0$ is a pure scale parameter. In contrast, the parameter $\beta$ has a decisive influence on the shape of the distribution $\Lambda$.

We now consider the Laplace-Stieltjes transform of $\Lambda$ for $\operatorname{Re}(s) \leqq 0 .(\operatorname{Re}(s)=0$ corresponds to the characteristic function.)

$$
\begin{aligned}
\lambda(s) & =\int_{0}^{\infty} e^{s y} d \Lambda(y) \\
& =\int_{0}^{\infty} e^{s y} d N\left(\frac{\log (\alpha y)}{\beta}\right)
\end{aligned}
$$

In order to continue $\lambda(s)$ analytically into the right $s$-halfplane we slightly rewrite $\lambda(s)$ for $s$ negative real and get

$$
\lambda(s)=\int_{0}^{\infty} e^{-u} d N\left(\frac{\log (\alpha u)}{\beta}-\frac{\log (-s)}{\beta}\right)
$$

Making the substitution $u=(I / \alpha) e^{-\beta y}$ we find

$$
\begin{aligned}
\lambda(s) & =\int_{0}^{\infty} e^{-(1 / \alpha) e^{-\beta \nu}} d N\left(y+\frac{\log (-s)}{\beta}\right) \\
& =\frac{I}{\sqrt{2 \pi}} \int_{-\infty}^{\infty} e^{-(1 / \alpha) e^{-\beta \nu}-1 / 2(y+(\log (-s)) / \beta)^{2}} d y .
\end{aligned}
$$


Since the last member of (2.3) represents an entire function of $\log (-s)$ we see that we have in (2.3) not only a representation of $\lambda(s)$ for $\operatorname{Re}(s) \leqq 0, s \neq 0$, but also an analytic continuation into the right halfplane if we avoid the point $s=0$ which is a branch point. If we avoid also the positive real axis we get in the remaining part of the plane, say $D$, a single-valued function. The boundary values of $\lambda(s)$ when we approach the positive real axis from above and from below, respectively, we denote by $\lambda+(x)$ and $\lambda-(x)$, respectively, where $x>0$.

From (2.3) we conclude that

$$
\begin{aligned}
& \lambda^{+}(x)=\frac{I}{\sqrt{2 \pi}} \int_{-\infty}^{\infty} e^{-(1 / \alpha) e^{-\beta y-1 / 2}\left(y+\frac{\log z}{\beta}-i \frac{\pi}{3}\right)^{2}} d y \\
& =\frac{e^{\pi^{2} /\left(2 \beta^{2}\right)}}{\sqrt{2 \pi}} \int_{-\infty}^{\infty} e^{-(1 / \alpha) e^{-\beta y-1 / 2}\left(y+\frac{\log x}{\beta}\right)^{2}+\frac{\pi i}{\beta}\left(y+\frac{\log x}{\beta}\right)} d y \\
& =\frac{e^{\pi^{2} /\left(2 \beta^{2}\right)}}{\sqrt{2 \pi}} \int_{-\infty}^{\infty} e^{-(x / \alpha) e^{-\beta \nu}-1 / 2 y^{2}+1(\pi / \beta) y} d y
\end{aligned}
$$

and

$$
\lambda^{-}(x)=\overline{\lambda^{+}(x)}=\frac{e^{\pi^{2} /\left(2 \beta^{2}\right)}}{\sqrt{2 \pi}} \int_{-\infty}^{\infty} e^{-(x / x) e^{-\beta v-1 / 2 y^{2}-i(\pi / \beta) y}} d y
$$

Taking real and imaginary parts we find

$$
\begin{aligned}
& \operatorname{Re} \lambda^{+}(x)=\frac{e^{\pi^{2}\left(2 \beta^{2}\right)}}{\sqrt{2 \pi}} \int_{-\infty}^{\infty} e^{-(x / x) e^{-\beta \nu-1 / 2 y^{2}}} \cos \frac{\pi y}{\beta} d y \\
& \operatorname{Im} \lambda^{+}(x)=\frac{e^{\pi^{2} /\left(2 \beta^{2}\right)}}{\sqrt{2 \pi}} \int_{-\infty}^{\infty} e^{-(x / x) e^{-\beta \nu-1 / 2 y^{2}}} \sin \frac{\pi y}{\beta} d y
\end{aligned}
$$

Note that

$$
\left|\lambda^{+}(x)\right|=\left|\lambda^{-}(x)\right| \leqq-\frac{e^{\pi^{2} /\left(2 \beta^{2}\right)}}{\sqrt{2 \pi}} \int_{-\infty}^{\infty} e^{-y^{2} / 2} d y=e^{\pi^{2} /\left(2 \beta^{2}\right)}
$$

Furthermore, the formula $(2.3)$ shows that $\lambda(s) \rightarrow 0$ uniformly in $\bar{D}$, the closure of $D$, when $|s| \rightarrow \infty$. It is also evident that $\lambda(s) \rightarrow$ I uniformly in $\bar{D}$ when $|s| \rightarrow 0$. In conjunction with $(2.8)$ these facts show that

$$
|\lambda(s)|<e^{\pi^{2} /\left(2 \beta^{2}\right)}
$$

for $s \in D$ (Phragmén-Lindelöf principle). 
However, it is easy to see directly that

$|\lambda(s)|<\frac{I}{\sqrt{2 \pi}} \int_{-\infty}^{\infty} e^{-1 / 2 R e}\left\{\left(y+\frac{\log (-s)}{\beta}\right)^{2}\right\} d y=e^{\frac{(\arg (-s))^{2}}{2 \beta^{2}}}<e^{\frac{\pi^{2}}{2 \beta^{2}}}$

when $-\pi<\arg (-s)<\pi$ i.e. for all points in $D$.

According to Cauchy's integral formula we have

$$
\lambda(s)=\frac{I}{2 \pi i} \int_{c} \frac{\lambda\left(s^{\prime}\right)}{s^{\prime}-s} d s^{\prime}
$$

where $s \in D$ and $C$ is a simple closed curve surrounding $s$.

Because of $\lambda(s)^{\prime} s$ properties when $|s| \rightarrow \infty$ and $|s| \rightarrow 0$ we may modify $C$ in such a way that we get

$$
\begin{aligned}
& \begin{aligned}
\lambda(s) & =\frac{I}{2 \pi i} \int_{0}^{\infty} \frac{\lambda^{+}(x)-\lambda^{-}(x)}{x-s}=\frac{I}{\pi} \int_{0}^{\infty} \frac{\operatorname{Im} \lambda^{+}(x)}{x-s} d x= \\
& =\int_{0}^{\infty} \frac{\operatorname{Im} \lambda^{+}(x) /(\pi x)}{I-s / x}
\end{aligned} \\
& \text { Defining } V^{\prime}(x)=\frac{\operatorname{Im} \lambda^{+}(x)}{\pi x}
\end{aligned}
$$

we find

$$
\lambda(s)=\int_{0}^{\infty} \frac{V^{\prime}(x) d x}{I-s / x}
$$

Using (2.7) we may write (2.II) in the form

$$
V^{\prime}(x)=\frac{e^{\pi^{2} /\left(2 \beta^{2}\right)}}{x \pi \sqrt{2 \pi}} \int_{-\infty}^{\infty} e^{-(x / \alpha) e^{-\beta y-1 / 2 y^{2}}} \sin \frac{\pi y}{\beta} d y
$$

In order to prove that

$$
\int_{0}^{\infty}\left|V^{\prime}(x)\right| d x<\beta / \pi \int_{0}^{\pi / \beta} e^{t^{2} / 2} d t<e^{\pi^{2} /\left(23^{3}\right)}
$$

we introduce

$$
Q(y)=\frac{\beta e^{\pi^{2} /\left(2 \beta^{2}\right)}}{\pi \sqrt{2 \pi}} \int_{-\infty}^{y} e^{-1 / 2 t^{2}} \sin \frac{\pi t}{\beta} d t
$$


Then we can rewrite (2.13):

$$
\begin{aligned}
V^{\prime}(x)=\frac{I}{\beta x} \int_{-\infty}^{\infty} e^{-(x / \alpha) e^{-\beta v}} d Q(y)= \\
=-\frac{I}{\alpha} \int_{-\infty}^{\infty} Q(y) e^{-\beta y} e^{-(x \mid \alpha) e^{-\beta v}} d y
\end{aligned}
$$

and get

$$
\begin{aligned}
\int_{0}^{\infty}\left|V^{\prime}(x)\right| d x<\frac{I}{\alpha} \int_{-\infty}^{\infty}|Q(y)| e^{-\beta y} d y \int_{0}^{\infty} e^{-(x / \alpha) e^{-\beta y}} d x \\
=\int_{-\infty}^{\infty}|Q(y)| d y
\end{aligned}
$$

However, it is easy, to rewrite $Q(y)$ in the following form (compare the derivatives!)

$$
Q(y)=-\frac{\beta}{\pi \sqrt{2 \pi}} e^{-y^{2} / 2} \int_{0}^{\pi / \beta} e^{t^{2} / 2} \cos (t y) d t
$$

Thus

$$
\int_{-\infty}^{\infty}|Q(y)| d y<\frac{\beta}{\pi} \int_{0}^{\pi / \beta} e^{t^{2} / 2} d t
$$

From (2.I7) and (2.I9) we get the asserted inequalities (2.I4).

It is now easy to invert (2.I2) to

$$
\begin{aligned}
\Lambda(y) & =\int_{0}^{\infty}\left(\mathrm{I}-e^{-x y}\right) V^{\prime}(x) d x \\
& =\int_{0}^{\infty}\left(\mathrm{I}-e^{-x y}\right) d V(x)
\end{aligned}
$$

where $V(x)$ is absolutely continuous and satisfies the conditions (i), (ii) and (iii) required in section $I$.

It is easy to see that $V^{\prime}(x)$ must have infinitely many zeros with a limit point in $\infty$. For that purpose we consider the successive derivatives of $\operatorname{Im} \lambda+(x)$. For convenience we also consider the derivatives of $\operatorname{Re} \lambda+(x)$. In fact we get from (2.6) and (2.7)

$$
\begin{aligned}
& \frac{d^{n}}{d x^{n}} \operatorname{Re} \lambda^{+}(x)=\lambda_{n} \operatorname{Re} \lambda^{+}\left(x e^{n \beta^{2}}\right) \\
& \frac{d^{n}}{d x^{n}} \operatorname{Im} \lambda^{+}(x)=\lambda_{n} \operatorname{Im} \lambda^{+}\left(x e^{n \beta^{2}}\right)
\end{aligned}
$$

where $\lambda_{n}=\frac{e^{n^{2} \beta^{2} / 2}}{\alpha^{n}}$ is the $n$th moment of $\Lambda(y)$. 
Since we know that $\operatorname{Re} \lambda+(0)=I ; \operatorname{Re} \lambda+(\infty)=0, \operatorname{Im} \lambda+(0)=0$, $\operatorname{Im} \lambda+(\infty)=0$ the relations $(2.2 \mathrm{I})$ and $(2.22)$ give some information about the shape of $\operatorname{Re} \lambda^{+}(x)$ and $\operatorname{Im} \lambda+(x)$. We thus conclude that $\operatorname{Re} \lambda^{+}(x)$ starts out from the value one at $x=0$, where all the derivatives to the right are positive, in fact they equal $\lambda_{n}$ for $n=\mathrm{I}, 2, \ldots$ In particular, $\operatorname{Re} \lambda+(x)$ near $x=0$ is increasing and convex. Since $\operatorname{Re} \lambda+(\infty)=0$ there must exist a point $x_{0}$ such that the derivative is zero in $x_{0}$. Then (2.2I) shows that $\operatorname{Re} \lambda+(x)$ has a zero at $x_{0} \cdot e^{\beta^{2}}$. Then there must exist a point $x_{1}>x_{0} e^{\beta^{2}}$ where the derivative is zero. This reasoning can be continued to show that there are infinitely many zeros tending to infinity. Clearly, the construction may be pursued in such a way that all zeros of $\operatorname{Re} \lambda+(x)$ are included. Note that $\operatorname{Re} \lambda+(x)$ must change sign infinitely many times.

A similar reasoning works for $\operatorname{Im} \lambda+(x)$. Since this function starts out from $\operatorname{Im} \lambda+(0)=0$ the present argument, however, does not exclude the possibility that the zeros also have a limit point at $x=0$. Note that all the derivatives at $x=0$ are zero.

From (2.II) we see that also $V^{\prime}(x)$ must have an infinity of zeros with $\infty$ as a limit point. Similarly $V^{\prime}(x)$ must change sign infinitely many times. Thus, i.a., $V(x)$ cannot be a distribution function. The fact that all the derivatives at $x=0$ of $\operatorname{Im} \lambda+(x)$ are zero entail that $V^{\prime}(x)$ has the same property.

Note also that all the absolute moments

$$
\int_{0}^{\infty} x^{n}\left|V^{\prime}(x)\right| d x, \quad n=0, I, 2, \ldots
$$

are finite. For $n=0$ we have just proved it. For $n>0$ it follows directly from (2.13).

The moments themselves are all zero for $n=\mathrm{I}, 2, \ldots$ but one for $n=0$. The latter fact is evident. The former fact can be followed from (2.13) by straight-forward integration. In fact we get

$$
\begin{aligned}
\int_{0}^{\infty} x^{n} V^{\prime}(x) d x & =\frac{e^{\pi^{2} /\left(2 \beta^{2}\right)}}{\pi \sqrt{2 \pi}} \int_{-\infty}^{\infty} e^{-1 / 2 y^{2}} \sin \frac{\pi y}{\beta} d y \int_{0}^{\infty} x^{n-1} e^{-x / \alpha e^{-\beta y}} d x \\
& =\frac{(n-I) ! \alpha^{n} e^{\pi^{2} /\left(2 \beta^{2}\right)}}{\pi \sqrt{2 \pi}} \int_{-\infty}^{\infty} e^{-1 / 2 y^{2}+n \beta y} \sin \frac{\pi y}{\beta} d y
\end{aligned}
$$


The integral in the last membrum equals.

$$
\begin{aligned}
e^{n^{2} \beta^{2} / 2} \int_{-\infty}^{\infty} e^{-1 / 2(y-n \beta)^{2}} \sin & \left(\frac{\pi(y-n \beta)}{\beta}+n \pi\right) d y= \\
& =(-I)^{n} e^{n^{2} \beta^{2 / 2}} \int_{-\infty}^{\infty} e^{-1 / 2 y^{2}} \sin \frac{\pi y}{\beta} d y=0
\end{aligned}
$$

Thus

$$
\int_{0}^{\infty} x^{n} V^{\prime}(x) d x=0, \quad n=\mathrm{I}, 2, \ldots
$$

However, a more rapid way to show (2.23) is to differentiate (2.20) $n$ times and to let $y=0$ observing that all the derivatives of $\Lambda(y)$ are zero at $y=0$.

\section{The Ruin Probabilities}

We now consider the ruin problem when the claim distribution is $\Lambda(y)$ and $K(t)$ is arbitrary. The initial risk reserve is assumed to be $u \geqq 0$ and the gross risk premium per time unit to be $c>0$.

We are interested in the probability $\Psi(u, t)$ that the risk reserve becomes negative somewhere in the time interval $(0, t]$. We try to get a formula for

$$
\bar{\Psi}(u, z)=\int_{0}^{\infty} e^{z t} d_{t} \Psi(u, t), \operatorname{Re}(z) \leqq 0
$$

in order to invert this formula by a numerical procedure.

In the same way as in our previous paper (Thorin and Wikstad (I973)) we find the formula

$$
\begin{array}{r}
\Psi(u, z)=\frac{\mathrm{I}}{A(0, z)} \int_{0} \frac{B(x, z) V^{\prime}(x) e^{-x u} d x}{k(z-c x)\left[\left(I / k(z-c x)-\operatorname{Re} \lambda^{+}(x)\right)^{2}+\left(\operatorname{Im} \lambda^{+}(x)\right)^{2}\right]} \\
+\sum_{f} g_{j}(z) e^{-u s_{z y}(z)}
\end{array}
$$

where $A(\cdot, \cdot), B(\cdot, \cdot), k(\cdot), g_{j}(\cdot), s_{2 j}(\cdot)$ are the usual auxiliary functions well known from our previous paper. 
In particular, $k(z)=\int_{0}^{\infty} e^{z t} d K(t)$ and $s_{2 j}(z)$ are the roots-lying in $D$ and such that $R e s_{2 j}(z)>0$-of the equation

$$
k\left(z-c s_{2 j}(z)\right) \lambda\left(s_{2 j}(z)\right)=\mathrm{I} \text {. }
$$

Furthermore,

$$
g_{j}(z)=\frac{B\left(s_{2 j}(z), z\right)}{A(0, z)\left[k\left(z-c s_{2 j}(z)\right) \lambda^{\prime}\left(s_{2 j}(z)-c k^{\prime}\left(z-c s_{2 j}(z)\right)\left(s_{2 j}(z)\right)\right] s_{2 j}(z)\right.}
$$

A check formula is $\bar{\Psi}(0, z)=I-\frac{I}{A(0, z)}$

For the case $K(t)=I-e^{-t}$, i.e. Poisson occurrences we get certain simplifications of the formulas in the following way.

$$
\begin{aligned}
\bar{\Psi}(u, z) & =\frac{z}{s_{1}(z)} \int_{0}^{\infty} \frac{\left(x-s_{1}(z)\right) V^{\prime}(x) e^{-x u} d x}{\left(\mathrm{I}+c x-z-\operatorname{Re} \lambda^{+}(x)\right)^{2}+\left(\operatorname{Im} \lambda^{+}(x)\right)^{2}} \\
& +\sum g_{j}(z) e^{-u s_{2 y}(z)}
\end{aligned}
$$

where

$$
\begin{gathered}
\mathrm{I}+c s_{2 j}(z)-z-\lambda\left(s_{2 j}(z)\right)=0, \quad \operatorname{Re}\left(s_{2 j}(z)\right)>0, \quad s_{2 j}(z) \in D \\
\mathrm{I}+c s_{1}(z)-z-\lambda\left(s_{1}(z)\right)=0, \quad \operatorname{Re}\left(s_{1}(z)\right) \leqq 0 \\
g_{j}(z)=z\left(\frac{\mathrm{I}}{s_{1}(z)}-\frac{\mathrm{I}}{s_{2 j}(z)}\right) \frac{\mathrm{I}}{\lambda^{\prime}\left(s_{2 j}(z)\right)-c}
\end{gathered}
$$

The check formula now reads

$$
\bar{\Psi}(0, z)=\mathrm{I}-\frac{z}{c s_{1}(z)}
$$

For the numerical illustrations we keep to the Poisson assumption $K(t)=\mathrm{I}-e^{-t}, t \geqq 0$ and thus use the formulas (3.6) through (3.I0). We invert the relation (3.I) using the same Piessens' algorithm (see Piessens (I 669 )) as we used in our previous paper. As to the lognormal distribution we fix the parameters to

$$
\begin{aligned}
& \beta=I .80 \\
& \alpha=e^{\beta^{2 / 2}}=e^{1.62}
\end{aligned}
$$


The choice of $\beta=1.80$ is taken from the paper by L.-G. Benckert and J. Jung to the Astin-colloquium in Essex, I973 (Benckert and Jung (1974)). These authors found the value $\beta=1.80$ in their investigation of the Swedish claim experience of fire insurance of stone dwellings reported I958-I969 (see their Table 3 Model A). The value $\alpha=e^{1.62}$ is chosen in order to get the mean amount one. (As pointed out above $\alpha$ is only a scale parameter.)

In our numerical illustrations we give a representative collection of values for $u=0$ by the use of formula (3.ro). For other values of $u$ we must use the formula (3.6). For the time being we have avoided such combinations of $c$ and $t$ which necessitates a search for roots $s_{2 f}(z)$ in the right halfplane. From the graph of $\operatorname{Re} \lambda+(x)$ it is possible to mark out the critical regions of $z$ for which such roots appear. If such critical $z$ 's must be used for a certain combination of $c$ and $t$ we have thus avoided the said combination. However, even if we are outside the critical regions but rather near one of them difficulties arise. In fact if a $s_{2 j}(z)$ lies very near the real axis, either effectively in $D$ or so to speak being on the way into $D$, the integrand in the integral term of (3.6) must be expected to have a "peak" which requires some caution in the numerical quadrature.

The critical $z$-regions for our choice of parameters can be characterized in the following way. For $c \geqq I$ I3 (about) there are, in principle, no critical regions. For $I<c<$ I.I3 (about) there is a certain $x$-interval $I_{c}$ in which $\operatorname{Re} \lambda+(x)$ lies above the straight line $I+c x$. The boundaries of the critical regions, one above the real axis and one below the same axis consist $I$ ) of the following curves

$$
\begin{aligned}
& \operatorname{Re}(z)=I+c x-\operatorname{Re} \lambda+(x) \\
& \operatorname{Im}(z)= \pm \operatorname{Im} \lambda+(x)
\end{aligned}
$$

where $x$ runs through $I_{c}$, and 2) of corresponding intervals on the imaginary axis.

From what we said above entails that also $z$ 's lying outside the critical regions but near them may be "critical" (even for $c$ lying sufficiently near but above I.I3).

It is possible to go around the indicated difficulties by modification of the integration line using the analytic continuation of the integrand. However, in the present work we have made no attempt in this direction. 


\section{The Approxinfant $\Lambda_{a}(y)$}

We have attempted to approximate $\Lambda(y)$ for $\beta=$ I.80 by a four or five terms combination of exponential distributions

$$
\begin{gathered}
\Lambda_{a}(y)=\mathrm{I}-\sum_{\cdot-1}^{m} a_{v} e^{-\alpha_{v} y} \\
0<\alpha_{j}<\alpha_{k} \text { for } j<k, \sum_{v-1}^{m} a_{v}=\mathrm{I}, m=4 \text { or } 5 .
\end{gathered}
$$

Similarly as in our previous paper we determine $\left\{a_{v}, \alpha_{v}\right\}_{1}^{m}$ as the solution of the system of equations

$$
\begin{aligned}
& \mathrm{I}-\Lambda(y)=\mathrm{I}-\Lambda_{a}(y) \\
& \int_{v}^{\infty}(\mathrm{I}-\Lambda(x)) d x=\int_{v}^{\infty}\left(\mathrm{I}-\Lambda_{a}(x)\right) d x \\
& y=0, \mathrm{IO}^{v}, v=0, \mathrm{I}, \ldots, m-2 .
\end{aligned}
$$

For the determination of $\Psi_{a}(u, t)$ in the Poisson case we use the relations

$$
\left.\begin{array}{l}
\bar{\Psi}_{a}(u, z)=\int_{0}^{\infty} e^{z t} d_{t} \Psi(u, t) \\
\bar{\Psi}_{a}(u, z)=\sum_{j=1}^{m} g_{j}(z) e^{-u s_{3 j}(z)}
\end{array}\right\}
$$

where $s_{2 j}(z)$ are the $m$ roots in the right halfplane of

$$
\begin{gathered}
I+c s-\lambda_{a}(s)=z \\
g_{j}(z)=\frac{\prod_{:-1}^{m}\left(\mathrm{I}-s_{2 j}(z) / \alpha_{v}\right)}{\prod_{\substack{i-1 \\
: \neq 1}}^{m}\left(\mathrm{I}-s_{2 j}(z) / s_{2 v}(z)\right)}
\end{gathered}
$$

Note that the number of terms in $\Lambda_{a}(y)$, necessary to get an acceptable approximation, depends on $\beta$. For "small" $\beta$ the number of terms may be prohibitive as may be inferred from the fact that $\Lambda(y)$ tends to $\varepsilon(y-I / \alpha)$ when $\beta \rightarrow 0$. In fact, an acceptable approximation of $\varepsilon(y-I / \alpha)$ by a linear combination of exponential distributions requires a "large" number of terms. $(\varepsilon(y-I / \alpha)$ is not representable in the form $(2.20)$. 
5. The Asymptotic Behavior of $\Psi(u)$ AND, $\Psi_{a}(u)$ FOR $u \rightarrow \infty$ As is very wellknown the asymptotic behavior $\left(c>\lambda_{1}\right)$ of $\Psi_{a}(u)$ is exponential.

In fact,

$$
\Psi_{a}(u) \sim C e^{-R u}, \quad u \rightarrow \infty
$$

where $C$ and $R$ are positive constants.

In contrast, $\Psi(u)$ has another asymptotic behavior:

$$
\Psi(u) \sim \frac{I}{c-\lambda_{1}} \int_{n}(I-\Lambda(y)) d y, \quad u \rightarrow \infty
$$

(Cf. Thorin (r974) pp. 97-98).

But we have

$$
\begin{aligned}
& \int(\mathrm{I}-\Lambda(y)) d y= \\
& \quad=\lambda_{1}\left(\mathrm{I}-N\left(\frac{\log (\alpha u)}{\beta}-\beta\right)-u\left(I-N\left(\frac{\log (\alpha u)}{\beta}\right)\right)\right.
\end{aligned}
$$

Wellknown asymptotic expressions for $\mathrm{I}-N(x)$ (see Cramér (I955) p. 38) now give for $u \rightarrow \infty$

$$
\begin{aligned}
\int_{*}^{\infty}(I-\Lambda(y)) & d y \sim \\
& \sim \frac{\beta^{3} \lambda_{1}}{\sqrt{2 \pi}} \frac{I}{\log (\alpha u) \log \left(\alpha e^{-\beta^{2}} u\right)} e^{-1 / 2\left(1 / \beta^{2}\right)\left(\log \left(\alpha e^{-3^{2}} u\right)\right)^{2}}
\end{aligned}
$$

and thus for $u \rightarrow \infty$

$\Psi(u) \sim \frac{\mathrm{I}}{c-\lambda_{1}} \frac{\beta^{3} \lambda_{1}}{\sqrt{2 \pi}} \frac{\mathrm{I}}{\log (\alpha u) \log \left(\alpha e^{\left.-\beta^{2} u\right)}\right.} e^{-1 / 2\left(1 / \beta^{2}\right)\left(\log \left(\alpha e-\beta^{2} u\right)\right)^{2}}$

\section{Numerical Methods}

The calculations are carried out in the same way as described in our previous joint paper (Thorin and Wikstad (I973)) except for the solution of the equation $I+c s-z=\lambda(s)$ in the left $s$-halfplane. The equation is written

$$
s=(I / c)(z-I+\lambda(s)) \equiv f(s)
$$


so that the familiar recursion formula $s^{(n+1)}=f\left(s^{(n)}\right)$ is obtained. As starting value $s^{(1)}=I / c(z-0.5)$ is chosen. No convergence problems have arisen.

The main integral in (3.2) requires calculations for a great number of points. The positive axis is divided into intervals by use of a logarithmic scale. In each interval a Gaussian quadrature based on twelve points is carried out.

The computer programs used are written in FORTRAN. The calculations are performed on a CDC 6600 .

\section{NuMERICAI REsults}

The $\left(a_{v}, \alpha_{v}\right)_{1}^{m}$ have been found to be $m=4$

\begin{tabular}{lll}
$v$ & \multicolumn{1}{c}{$a_{v}$} & \multicolumn{1}{c}{$\alpha_{v}$} \\
$\mathrm{I}$ & $0.0009872 \mathrm{IOI}$ & $0.0 \mathrm{I} 2878 \mathrm{I} 7$ \\
2 & $0.035409 \mathrm{I}$ & $0.0972492 \mathrm{I}$ \\
3 & $0.2855 \mathrm{I} 4 \mathrm{I}$ & 0.6569755 \\
4 & 0.6780897 & 5.440050
\end{tabular}

$m=5$

\begin{tabular}{lll}
$v$ & \multicolumn{1}{c}{$a_{v}$} & \multicolumn{1}{c}{$\alpha_{v}$} \\
$I$ & 0.000007137059 & $0.00 I 887727$ \\
2 & $0.00 I I 73100$ & 0.01480705 \\
3 & 0.03587 I77 & $0.09955^{8} 433$ \\
4 & $0.28543 I I$ & 0.6601540 \\
5 & 0.6775 I69 & 5.445927
\end{tabular}

All other results are presented in the tables.

\section{CONCLUding REMARKS}

This paper has been written as a part of the work carried out by the Swedish committee for the practical applications of the risk theory. Of the two authors Thorin is responsible for the sections I-5 and Wikstad for the sections 6-7 including the attached tables. 
Tables showing numerical values of min probabilities

\section{TABLE I}

Claim d.f.: lognormal with parameters $\beta=1.80, \alpha=e^{1.61}$

Interclaim time d.f.: $K(t)=\mathrm{I}-e^{-t}$

(Empty places correspond to "critical" regions)

$\begin{array}{lrllllllll} & u \quad c=1.05 & 1.10 & 1.15 & 1.20 & 1.25 & 1.30 & 2.00 \\ T=100 & 0 & .82192 & .79870 & .77571 & .75314 & .73115 & .70982 & .48805 \\ & 100 & .03701 & .03461 & .03246 & .03054 & .02881 & .02726 & .01525 \\ & 1000 & .00011 & .00011 & .00011 & .00011 & .00011 & .00011 & .00010 \\ T=1000 & 0 & .91556 & .88534 & .85407 & .82301 & .79293 & .76423 & .49967 \\ & 100 & . & . . & .16740 & .13511 & .11123 & .09334 & .02483 \\ & 1000 & . & . & .00108 & .00100 & .00093 & .00087 & .00045 \\ & & & & & & & & & \\ 150 & 0 & .95238 & .90909 & .86957 & .83333 & .80000 & .76923 & .50000 \\ & 100 & .55074 & .34395 & .23573 & .17309 & .13384 & .10765 & .02535 \\ & 1000 & .04199 & .01099 & .00574 & .00384 & .00288 & .00230 & .00060 \\ & 10000 & .00008 & .00004 & .00002 & .00002 & .00001 & .00001 & .00000\end{array}$

TABLE 2

Claim d.f.: $\Lambda_{a}(y)=\dot{\sum}_{\cdot=1} a_{v}\left(\mathrm{I}-e^{-x_{v} y}\right)$

Interclaim time d.f.: $K(t)=\mathrm{I}-e^{-t}$

$\begin{array}{lrllllllll} & u & c=1.05 & 1.10 & 1.15 & 1.20 & 1.25 & 1.30 & 2.00 \\ T=100 & 0 & .82617 & .80295 & .77986 & .75711 & .73487 & .71324 & .48869 \\ & 100 & .03483 & .03286 & .03112 & .02956 & .02817 & .02691 & .01664 \\ & 1000 & .00000 & .00000 & .00000 & .00000 & .00000 & .00000 & .00000 \\ T=1000 & & & & & & & & & \\ & 0 & .91738 & .88722 & .85601 & .82497 & .79487 & .76609 & .49997 \\ & 100 & .26749 & .20936 & .16664 & .13514 & .11163 & .09382 & .02439 \\ & 1000 & .00004 & .00003 & .00002 & .00001 & .00001 & .00001 & .00000 \\ & & & & & & & & \\ & 0 & .95238 & .90909 & .86957 & .83333 & .80000 & .76923 & .50000 \\ & 100 & .53669 & .32960 & .22367 & .16340 & .12609 & .10140 & .02439 \\ & 1000 & .01688 & .00122 & .00022 & .00006 & .00003 & .00001 & .00000 \\ & 10000 & .00000 & .00000 & .00000 & .00000 & .00000 & .00000 & .00000\end{array}$




\begin{tabular}{|c|c|c|c|c|c|c|c|c|}
\hline & & & & {$[A B L E=$} & & & & \\
\hline Clain & $\mathrm{m} d$. & $\therefore \Lambda_{a}(y$ & $=\sum_{n=1}^{s}$ & $a_{y}(\mathrm{I}$ & $\left.e^{-x_{v} y}\right)$ & & & \\
\hline Inter & rclaim & time & 1.f.: $K$ & t) $=I$ & $-e^{-t}$ & & & \\
\hline & $u$ & $=1.05$ & 1.10 & I.I 5 & $\mathrm{I} .20$ & 1.25 & 1.30 & 2.00 \\
\hline$T=100$ & o & .82587 & .80263 & $.7795+$ & .75679 & $.73+55$ & .71294 &.+8861 \\
\hline & 100 & $.03+97$ & .03292 & .03 I I I & .02949 & .02803 & .02671 & .01595 \\
\hline & 1000 & $.0001 \mathrm{I}$ & $.000 \mathrm{II}$ & $.000 \mathrm{II}$ & $.0001 \mathrm{I}$ & $.0001 \mathrm{I}$ & $.000 I I$ & .00010 \\
\hline$r=1000$ & 0 & .01706 & .88676 & .85540 &.$S 2423$ & $.7940 \mathrm{I}$ & .765 I 6 & .49968 \\
\hline & IOO & $.265 \mathrm{II}$ & .20635 & .06323 & .13159 & .10817 & .09058 & .02397 \\
\hline & 1000 & .00118 & .00 II & .00104 & .00098 & .00093 & .00089 & .00050 \\
\hline$\infty$ & o & $.9523^{8}$ & .90909 & .86957 & .83333 & .80000 & .76923 & .50000 \\
\hline & 100 & $.537^{84}$ & .33082 & $.2247 \mathrm{I}$ & .16425 & .12677 & . 10195 & $.024+7$ \\
\hline & 1000 & $.03+40$ & $.0094 \mathrm{I}$ & .00520 & $.0035^{8}$ & .00273 & $.0022 \mathrm{I}$ & .00060 \\
\hline & 10000 & .00000 & .00000 & .00000 & .00000 & .00000 & .00000 & .00000 \\
\hline
\end{tabular}

\section{REFERENCES}

AIrchisos, J. and Browx, J. A. C. 1957. The lognormal distribution with special reference to its uses in economics. Cambridge University Press.

BENCKERT, L.-G. and JUNG, J. In74. Statistical models of claim distributions in fire insurance. Astin Bullctin, $I^{-} I I I, \mathrm{x}-25$.

Cranér, H. 1955. Collective risk theory. Jubilee rolume of Forsaknngsaktiebolaget Skandia.

Piessens, R. 1969. New quadrature formulas for the numerical inversion of the Laplace transform. BIT, 9. 35 I-36r.

SEAL, H. L. I969. Simulation of the ruin potential of nonlife insurance companies. Transactions of the Society of Actuares, XXI, 563-j85.

THORIN, O. 1974. On the asymptotic behavior of the ruin probability for an infinite period when the epochs of claims form a renewal process. Scand. Actuarial Journal, \&x-99.

Thorin, O. and Wikstad, N. r973. Numerical evaluation of ruin probabilities. Astin Bulletin, III, I37-1 53 .

Thyrion. P. I964. Les lois exponentielles composées. Assuciation Royale des Actuaires Belges Bulletin, 62, 35-44. 